\title{
Synthesis of 3-Aminohydantoinyl-1,2-benzothiazine Derivatives
}

\author{
Myung-Sook Park, Eun-Sung Chang, Myung-Sook Lee, and Soon-Kyoung Kwon* \\ College of Pharmacy, Duksung Women's Universitv, Seoul 132-714, Korea \\ Received August 24, 2002
}

Key Words : 3-Aminohydantoiny l-1 2-benzothiaziness. 3-Aminohỵdantoins

Since 1.2-benzothiazine was synthesized for the first time by Braun. ${ }^{3}$ several synthetic methods of 4-hydroxy-1.2benzothiazines were developed by Abe et $\alpha f^{\dot{*}}$ and these compounds have been investigated continuously because of biological and pharmacological properties. Recently. several 1.2-benzothiazines such as droxicam. ${ }^{3}$ ampiroxicam. ${ }^{4}$ meloxicam. ${ }^{5}$ lomoxicam ${ }^{6}$ have been developed as nonsteroidal anti-inflammatory drugs (NSAIDs), years after piroxicam was introduced on the market in $1971 .^{7}$

Previously, we have reported the syntheses of some new 1.2-benzothiazine derivatives $s^{8.9}$ and 1-amino-2-thioxohy dantoin. ${ }^{\text {to }} \mathrm{By}$ the application of these methods. 1.2-benzothiazine derivatives with 3-aminohydantoin moiety were prepared. Here. we report the synthesis of several new 3aminohydantoinyl-1.2-benzothiazine derivatives and propose an another mechanism of the cyclization to the hydantoins

3-Aminohydantoins 3a-d were prepared through cyclization of the condensation products $2 \mathrm{a}-\mathbf{d}$ that were formed by heating amino acids and tert-butyl carbazate in quinoline according to the method of Lalezari. "] Even though L-amino acids were applied. the products were racemized. We used the quinoline that was freshly distilled under vaccum after standing with potassium hydroxide for a few days because the success of this reaction depends on the water content of quinoline. The yields of compounds (3a-d) were about 86$96 \%$ and those were identified using NMR. Because tertbutyl carbazate was often sublimated as white solid in the condenser. it was important to react the amino acids with excess (twofold mol \%) tert-butyl carbazate. The generated byproduct. tert-butyl alcohol was removed during refluxing over the Dean-Stark apparatus.

Compounds 7a-c were synthesized according to the method as described in the previous report. ${ }^{8} 6-H a l o g e n ~(C l$. $\mathrm{Br}$ ) substituted saccharins 5b-c were synthesized through the process of chlorosulfonation, ammonolysis and oxidation of $p$-halotoluene. 1,2-Benzothiazine derivatives 7a-c were sỵnthesized through Gabriel-Colman rearrangement after condensation of sodium halo (or $\mathrm{H}$ ) saccharin with methyl clloroacetate. 7-Halo (or $\mathrm{H}$ )-1,2-benzothiazine-3-carboxamide derivatives 8a-j were sy'nthesized through the condensation of 7-halo(or H)-4-hydroxy-2H-1.2-benzothiazine-3-carboxylic acid methyl ester 1.2-dioxides (7a-c) with 3-amino-5alky limidazolidine-2.4-diones (3a-d) in xylene as shown in Scheme 2

The reaction mechanism of the formation of the 3-amino-
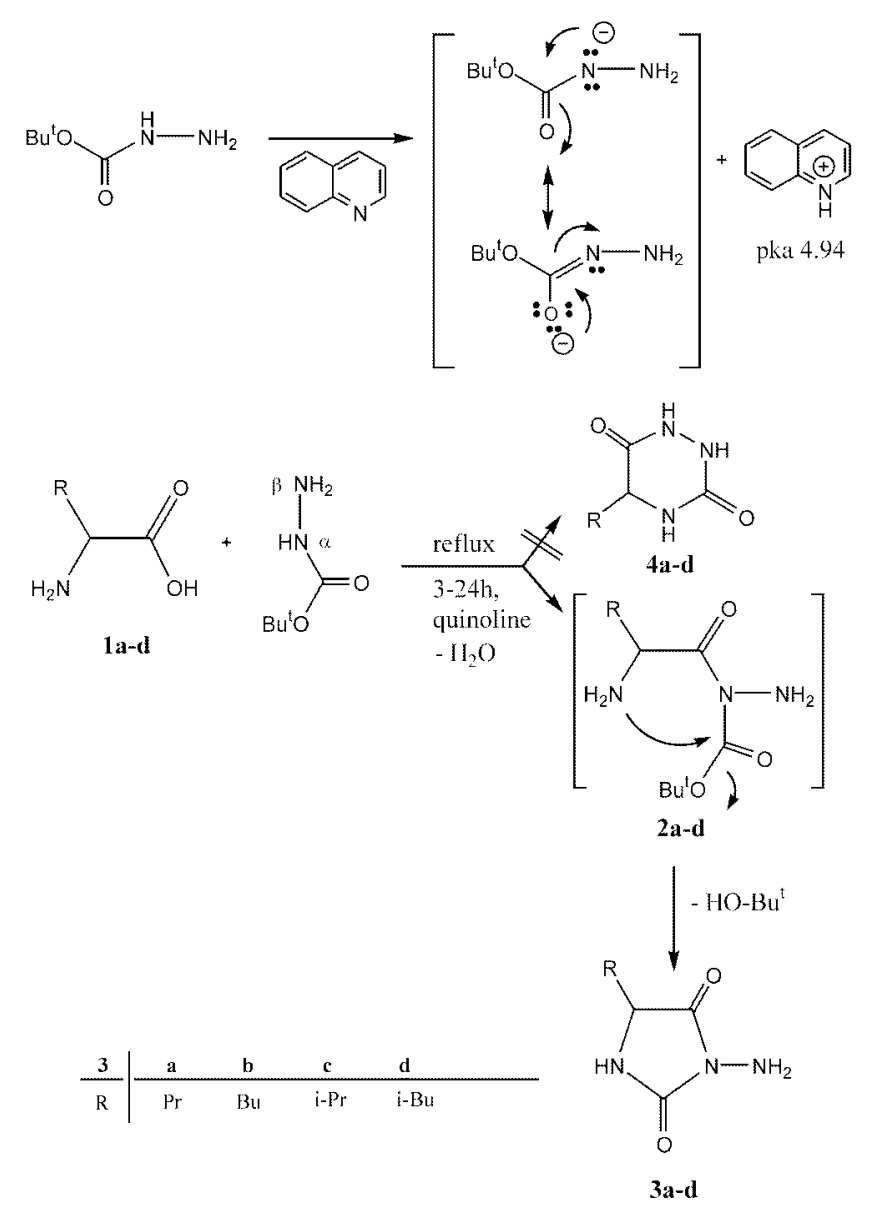

Scheme 1

hydantoins (3a-d) involves the amidation and cyclization between $\alpha$-amino acid and tert-butyl carbazate. One molecule of tert-butanol is generated from intermediate $2 \mathrm{a}-\mathbf{d}$ by the intramolecular nucleophillic attack of amino group to the electron deficient carbonyl carbon of ester. In general. compounds 3a-d can be easily formed because tert-butoxyl group is very good leaving group. The cyclization products of amino acids and tert-butyl carbazate were found to be 3 aminohydantoins (3a-d) rather than hexalyydro-1,2,4-triazine-3,6-diones (4a-d)

Even though Lalezari ${ }^{1]}$ proposed a possible reaction mechanism of the formation of the 3-aminohydantoin. we propose another possible mechanism outlined in Scheme 1. According to our opinion. the driving force of this reaction is the nucleophilic attack of $\alpha$-nitrogen of tert-butyl carbazate 
<smiles>[X]c1ccc2c(c1)S(=O)(=O)N([Y6])C2=O</smiles><smiles>CC(=O)CC1CCCCC1</smiles><smiles>[X]c1ccc2c(c1)S(=O)(=O)N(CC(=O)OC)C2=O</smiles>
$\mid \begin{aligned} & \mathrm{CH}_{3} \mathrm{ONa} \\ & \mathrm{CH}_{3} \mathrm{OH} \\ & \text { reflux, ih }\end{aligned}$<smiles>[X]c1ccc2c(c1)S(=O)(=O)NC(C(C)=O)=C2O</smiles><smiles>[R]C1NC(=O)N(N)C1=O</smiles><smiles>[X]c1ccc2c(c1)S(=O)(=O)NC(C(=O)NN1C(=O)NC([R])C1=O)=C2O</smiles>

\begin{tabular}{c|ccccc}
$\mathbf{8}$ & $\mathbf{a}$ & $\mathbf{b}$ & $\mathbf{c}$ & $\mathbf{d}$ & $\mathbf{e}$ \\
\hline $\mathrm{X}$ & $\mathrm{H}$ & $\mathrm{Cl}$ & $\mathrm{Cl}$ & $\mathrm{Cl}$ & $\mathrm{Cl}$ \\
$\mathrm{R}$ & $\mathrm{i}-\mathrm{Pr}$ & $\mathrm{Pr}$ & $\mathrm{Bu}$ & $\mathrm{i}-\mathrm{Pr}$ & $\mathrm{i}-\mathrm{Bu}$ \\
$\mathbf{8}$ & $\mathbf{f}$ & $\mathbf{g}$ & $\mathbf{h}$ & $\mathbf{i}$ & \\
\hline $\mathrm{Br}$ & $\mathrm{Br}$ & $\mathrm{Br}$ & $\mathrm{Br}$ & \\
$\mathrm{R}$ & $\mathrm{Pr}$ & $\mathrm{Bu}$ & $\mathrm{i}-\mathrm{Pr}$ & $\mathrm{i}-\mathrm{Bu}$
\end{tabular}

Scheme 2

to the carbon of carboxyl group of amino acid

The proton of $\alpha$-nitrogen of tert-butyl carbazate is more acidic than the proton of $\beta$-nitrogen because of vicinal carbonyl group. The $\mathrm{pKa}$ of quinoline as solvent is 4.94 similar to 5.23 of pyridine. The basic quinoline is easier to accept a proton of $\alpha$-nitrogen than a proton of $\beta$-nitrogen. As a result. the $\alpha$-nitrogen of tert-butyl carbazate attacks carboxyl carbon of the amino acid. After then the primary amino group of unstable intermediate 2a-d attacks intramolecularly to the carbonyl carbon of ester and 3-aminohydantoins 3a-d are formed. This cyclization method from $\alpha$-amino acids could be applicable to the preparation of various hydantoins.

We previously reported that 1 -aminohydantoins ${ }^{10}$ and 3.5substituted-1-aminolyydantoins ${ }^{12}$ were prepared by the condensation of aralhylthiocyanate with ethyl hy'drazinoacetate hydrochloride (or ethyl 2-hydrazinopropionate monohydroclloride) in the presence of triethylamine. The intermediates. $\mathrm{N}$-amino-N-ethoxycarbony lmethyl-N'-aralky'thioureas . which were formed during the reaction and could be transformed into the appropriate 1-aminolydantoins, were isolated and characterized. This intermediates were formed because the proton of $\alpha$-nitrogen(secondary amino group) of $\mathrm{N}$-amino-N-ethoxy carbony lmetlyy l-N'-aralkylthioureas is more acidic than the proton of $\beta$-nitrogen (primary amino group). In this reaction. triethylamine was applied as a base and 1 -aminolydantoins (5-membered ring) could be exclusively obtained instead of hexahydro-1,2.4-triazine-3,6diones (6-membered ring). In principle, this reaction is same as the 3-aminohydantoin formation from $\alpha$-amino acids (1ad) and tert-butyl carbazate

But Lalezari reported that primary amino group $(\beta-$ nitrogen) initially attacks to the electron deficient carbosyl carbon of amino acid ${ }^{11}$ and the intermediate is cyclized to an unstable diaziridinone. And then the primary amino group of intermediate attacks the carbonyl carbon of the diaziridinone.

Finally, novel 7-halo-4-hydrosy-2H-N-(5-alkyl-3-aminohydantoinyl)-1,2-benzothiazine-3-carboxamide 1.l-dioxides 8a-i were synthesized through the condensation of related 1.2-benzothiazine and 3-aminolydantoins. Conpounds 8a-i were evaluated for analgesic and anti-inflammatory activities and exhibit a good activities.

\section{Experimental Section}

Chemicals were supplied by Aldrich, Signa, Merck. and Tohyo Kasei. Melting points were determined in open capillary tubes on a Büchi 535 melting point apparatus and uncorrected. The NMR spectra were recorded using Gemini Varian $300 \mathrm{MHz}$ NMR spectrometer. Chemical shift values were reported in parts per million on the scale in deuterioclloroform or dimethyl-d $\mathrm{d}_{6}$ sulfoxide with tetramethylsilane as the intenal standard. The NMR spin multiplicities were indicated by the symbols: $\mathrm{s}$ (singlet), $\mathrm{d}$ (doublet), $\mathrm{t}$ (triplet), $\mathrm{q}$ (quartet) and $m$ (multiplet). IR spectra were recorded on a Perkin-Elmer 16F PC FT-IR spectrometer using $\mathrm{NaCl}$ discs.

General procedure for the synthesis of 5-alkyl-3aminohydantoin derivatives (3a-d). L-Anino acid (0.036 mole) and tert-butyl carbazate $(0.072 \mathrm{~mole})$ were refluxed for about $5-10 \mathrm{hrs}$ in distilled quinoline $(80 \mathrm{~mL})$ using DeanStark apparatus until the disappearance of the starting material. The reaction mixture was cooled and ether $(90 \mathrm{~mL})$ was poured into the mixture. The precipitated solid by adding slowly with $n$-hexane $(270 \mathrm{~mL})$ was filtered and recrystallized from 2-propanol-water.

Yield $86-96 \%$

General procedure for the synthesis of 7-substituted (or nonsubstituted) 4-hydroxy-2H-1,2-benzothiazine-3carboxylic acid methỵl ester 1,1-dioxide derivatives (7ac). A solution of sodium methoxide was prepared from sodium $(1.065 \mathrm{~mol})$ in absolute methanol. To the cooled solution in an ice-bath 7 a-c $(0.15 \mathrm{~mol})$ was added immediately as powder. Color changes from yellow to orange. After a few minutes. the mixture was refluxed for $1 \mathrm{~h}$. The orange slurry was poured into ice-cold concentrated hydrochloric acid $(100 \mathrm{~mL})$. The mixture was cooled in an ice bath. The 
precipitate was filtered off, washed with water and the residue was recrystallized from diluted methanol.

Yield 55-85\%

General procedure for the synthesis of 3-aminohydantoinyl-1,2-benzothiazine derivatives (8a-i). 4-Hydroxy$2 \mathrm{H}-1$.2-benzothiazine-3-carboxylic acid methyl ester 1,2dioxides $(0.004$ mole) and 5-alkyl-3-aminohydantoin $(0.0056$ mole) were refluxed for about $24 \mathrm{hrs}$ in sylene $(15 \mathrm{~mL})$ in a Dean-stark apparatus until the disappearance of the starting material. The reaction misture was allowed to cool to room temperature. evaporated to decrease volume of $x y$ lene to $1 / 3$. The cooled reaction mixture was filtered to yield a pale yellow solid after washing with ethyl acetate. The crude solid was recrystallized from methanol.

4-Hydroxy-2H-N-(5-isopropyl-3-aminohydantoinyl)-1,2benzothiazine-3-carboxamide 1,1-dioxide 8a. Yield $1.11 \mathrm{~g}$ (73\%). mp $287-289^{\circ} \mathrm{C}$ (dec). Recrystal solvent: methanol: IR $\left(\mathrm{NaCl}, \mathrm{cm}^{-1}\right) 3370(\mathrm{NH}), 3130(\mathrm{NH}) .302 \mathrm{l}$ (aromatic). $1741(\mathrm{CO}), 1377\left(\mathrm{SO}_{2}\right) ;{ }^{1} \mathrm{H}$ NMR (DMSO-d 6 ) $\delta 11.03$ (s. $1 \mathrm{H}, \mathrm{NH}), 9.83(\mathrm{~s}, \mathrm{lH}, \mathrm{NH}), 8.58(\mathrm{~s}, \mathrm{lH}, \mathrm{OH}), 7.88-7.8 \mathrm{l}(\mathrm{m}$. $\left.4 \mathrm{H}, \mathrm{C}_{6} \mathrm{H}_{4}\right), 4.2 \mathrm{l}(\mathrm{d} . J=5.4 \mathrm{~Hz} . \mathrm{lH} . \mathrm{CH}) .2 .12 \cdot 2.09(\mathrm{~m}, \mathrm{lH}$. $\mathrm{CH}) .1 .02\left(\mathrm{~d}, J=6.9 \mathrm{~Hz}, 3 \mathrm{H} . \mathrm{CH}_{3}\right), 0.79(\mathrm{~d} . J=6.9 \mathrm{~Hz} .3 \mathrm{H}$. $\mathrm{CH}_{3}$ ).

7-Chloro-4-hydroxy-2H-N-(5-propyl-3-aminohydantoinyl)-1,2-benzothiazine-3-carboxamide 1,1-dioxide 8b. Yield $51 \%$, mp $286-288^{\circ} \mathrm{C}$ (dec), Recrystal solvent: methanol; IR $\left(\mathrm{NaCl}, \mathrm{cm}^{-1}\right) 3240(\mathrm{NH}), 3130(\mathrm{NH}) .3010$ (aromatic). 1720 (CO). 1380 ( $\left.\mathrm{SO}_{2}\right) .730$ (C.Cl): ${ }^{1} \mathrm{H}$ NMR (DMSO-d 6 ) $\delta$ 11.07 (s. $1 \mathrm{H} . \mathrm{NH}$ ). $8.6 \mathrm{l}$ (s. $1 \mathrm{H}, \mathrm{OH}$ ) $8.05-7.91$ (m. $3 \mathrm{H}$. $\mathrm{C}_{6} \mathrm{H}_{3} \mathrm{Cl}$ ), 4.30 (t. $J=6.1 \mathrm{~Hz}, 1 \mathrm{H}$. CH). $1.75-1.57$ (m. $2 \mathrm{H}$. $\left.\mathrm{CH}_{2}\right) .1 .42-1.40\left(\mathrm{~m} .2 \mathrm{H}, \mathrm{CH}_{2}\right), 0.92\left(\mathrm{t}, J=7.1 \mathrm{~Hz}, 3 \mathrm{H} . \mathrm{CH}_{3}\right)$.

7-Chloro-4-hydroxy-2H-N-(5-butyl-3-aminohydantoinyl)1,2-benzothiazine-3-carboxamide 1,1-dioxide 8c. Yield 56 $\%$, mp $266-268{ }^{\circ} \mathrm{C}$ (dec). Recrystal solvent: methanol: IR $\left(\mathrm{NaCl} \mathrm{cm}^{-1}\right) 3190(\mathrm{NH}) .3020$ (aromatic), 1720 (CO). 1380 $\left(\mathrm{SO}_{-}\right) .730(\mathrm{C}-\mathrm{Cl}):{ }^{1} \mathrm{H}$ NMR (DMSO-d $) \delta 11.05$ (s, $1 \mathrm{H}$. NH). $8.60(\mathrm{~s}, \mathrm{lH} . \mathrm{OH}) .8 .07-7.95\left(\mathrm{~m}, 3 \mathrm{H}, \mathrm{C}_{6} \mathrm{H}_{3} \mathrm{Cl}\right) .4 .30$ (t. $J$ $=5.8 \mathrm{~Hz} . \mathrm{lH} . \mathrm{CH}), 1.73-1.72\left(\mathrm{~m} .2 \mathrm{H} . \mathrm{CH}_{2}\right), 1.64-159(\mathrm{~m}$. $\left.2 \mathrm{H} . \mathrm{CH}_{2}\right) .1 .32-1.27\left(\mathrm{~m} .2 \mathrm{H} . \mathrm{CH}_{2}\right) .0 .87$ (t. $J=6.8 \mathrm{~Hz}, 3 \mathrm{H}$. $\mathrm{CH}_{3}$ ).

7-Chloro-4-hydroxy-2H-N-(5-isopropyl-3-aminohydantoinyl)-1,2-benzothiazine-3-carboxamide 1,1-dioxide 8d. Yield $83 \%$. mp $272-274^{\circ} \mathrm{C}(\mathrm{dec})$. Recrystal solvent: methanol: IR $\left(\mathrm{NaCl} . \mathrm{cm}^{-1}\right) 3300(\mathrm{NH}) .3040$ (aromatic). $1725(\mathrm{CO})$. $1380\left(\mathrm{SO}_{2}\right) .725(\mathrm{C}-\mathrm{Cl}):{ }^{1} \mathrm{H}$ NMR (DMSO-d $) \delta 11.07$ (s. lH. NH). 8.58 (s. $1 \mathrm{H} . \mathrm{OH}$ ) $8.07-7.95$ (m. $3 \mathrm{H}, \mathrm{C}_{6} \mathrm{H}_{3} \mathrm{Cl}$ ) 4.20 (d. $J=5.9 \mathrm{~Hz}, 1 \mathrm{H}, \mathrm{CH}), 2.12-2.10(\mathrm{~m}, 1 \mathrm{H} . \mathrm{CH}) .1 .02(\mathrm{~d} . J=$ $\left.6.9 \mathrm{~Hz}, 3 \mathrm{H} . \mathrm{CH}_{3}\right) .0 .89$ (d. $J=6.9 \mathrm{~Hz} .3 \mathrm{H} . \mathrm{CH}_{3}$ ).

7-Chloro-4-hydroxy-2H-N-(5-isobutyl-3-aminohydantoinyl)-1,2-benzothiazine-3-carboxamide 1,1-dioxide 8e. Yield $80 \%$ mp 283-285 ${ }^{\circ} \mathrm{C}$ (dec). Recrystal solvent: methanol: IR $\left(\mathrm{NaCl} . \mathrm{cm}^{-1}\right) 3295(\mathrm{NH}) .3020$ (aromatic). $1720(\mathrm{CO})$. $1380\left(\mathrm{SO}_{2}\right) .720(\mathrm{C}-\mathrm{Cl}):{ }^{1} \mathrm{H}$ NMR (DMSO-d $) \delta 11.04$ (s. lH. NH). 8.68 (s. 1H. OH) $8.09-7.95$ (m. $3 \mathrm{H} . \mathrm{C}_{6} \mathrm{H}_{3} \mathrm{Cl}$ ) 4.30 (t. $J=5.4 \mathrm{~Hz}, 1 \mathrm{H}, \mathrm{CH}$ ), $1.86-1.82\left(\mathrm{~m}, 2 \mathrm{H}, \mathrm{CH}_{2}\right.$ ). 1.64-1.47 (m. $2 \mathrm{H} . \mathrm{CH}_{2}$ ). 0.93 (d. $J=7.2 \mathrm{~Hz}, 6 \mathrm{H}, \mathrm{CH}_{3} \times 2$ ).

7-Bromo-4-hydroxy-2H-N-(5-propyl-3-aminohydantoin- yl)-1,2-benzothiazine-3-carboxamide 1,1-dioxide 8f. Yield $73 \%$. mp 288-291 ${ }^{\circ} \mathrm{C}$ (dec). Recrystal solvent: methanol: IR $\left(\mathrm{NaCl}, \mathrm{cm}^{-1}\right) 3240(\mathrm{NH}) .3130(\mathrm{NH}), 3010$ (aromatic), 1720 (CO), $1380\left(\mathrm{SO}_{2}\right), 730(\mathrm{C}-\mathrm{Br}),{ }^{1} \mathrm{H}$ NMR (DMSO-d 6 ) $\delta 11.07$ $(\mathrm{s}, 1 \mathrm{H}, \mathrm{NH}), 8.61$ (s. $\mathrm{lH}, \mathrm{OH}) .8 .09-7.91\left(\mathrm{~m} .3 \mathrm{H}, \mathrm{BrC}_{6} \mathrm{H}_{3}\right.$ ), $4.30(\mathrm{t}, J=5.4 \mathrm{~Hz}, \mathrm{lH} . \mathrm{CH}) .1 .75-1.57\left(\mathrm{~m}, 2 \mathrm{H} . \mathrm{CH}_{2}\right), 1.42-$ 1.37 (m. $\left.2 \mathrm{H} . \mathrm{CH}_{2}\right) .0 .92$ (t. $J=7.2 \mathrm{~Hz}, 3 \mathrm{H}, \mathrm{CH}_{3}$ ).

7-Bromo-4-hydroxy-2H-N-(5-butyl-3-aminohydantoinyl)-1,2-benzothiazine-3-carboxamide 1,1-dioxide 8g. Yield $64 \%$. mp $286-287^{\circ} \mathrm{C}$ (dec). Recrystal solvent: methanol: IR $\left(\mathrm{NaCl}, \mathrm{cm}^{-1}\right) 3190(\mathrm{NH}) .3130(\mathrm{NH}), 3010$ (aromatic), 1720 (CO), $1380\left(\mathrm{SO}_{2}\right), 730(\mathrm{C}-\mathrm{Br}) ;{ }^{1} \mathrm{H}$ NMR (DMSO-d 6 ) $\delta 11.09$ $(\mathrm{s}, \mathrm{lH}, \mathrm{NH}), 8.60$ (s. $\mathrm{lH}, \mathrm{OH}) .8 .07-7.95\left(\mathrm{~m} .3 \mathrm{H}, \mathrm{BrC}_{6} \mathrm{H}_{3}\right)$, $4.30(\mathrm{t}, J=5.4 \mathrm{~Hz}, 1 \mathrm{H} . \mathrm{CH}) .1 .79-1.55\left(\mathrm{~m}, 2 \mathrm{H} . \mathrm{CH}_{2}\right), 1.32-$ 1.29 (m. $\left.2 \mathrm{H} . \mathrm{CH}_{2}\right) .0 .87$ (t. $J=7.2 \mathrm{~Hz}, 3 \mathrm{H}, \mathrm{CH}_{3}$ ).

7-Bromo-4-hydroxy-2H-N-(5-isopropyl-3-aminohydantoinyl)-1,2-benzothiazine-3-carboxamide 1,1-dioxide $8 \mathrm{~h}$. Yield $86 \%$. mp $283-285^{\circ} \mathrm{C}$ (dec), Recrystal solvent: methanol: IR $\left(\mathrm{NaCl}, \mathrm{cm}^{-1}\right) 3300(\mathrm{NH}), 3130(\mathrm{NH}), 3010$ (aromatic), $1725(\mathrm{CO}), 1380\left(\mathrm{SO}_{2}\right) .725(\mathrm{C} \cdot \mathrm{Br}) .{ }^{1} \mathrm{H}$ NMR (DMSO-d 6$) \delta$ 11.07 (s, lH. NH), 8.58 (s. 1H. OH), $8.10-7.92$ (m, 3H, $\left.\mathrm{BrC}_{6} \mathrm{H}_{3}\right) .4 .12(\mathrm{~d}, J=5.4 \mathrm{~Hz}, \mathrm{lH}, \mathrm{CH}), 2.19-2.06(\mathrm{~m} . \mathrm{lH}$. CH), 1.02 (d. $J=7.2 \mathrm{~Hz}, 3 \mathrm{H}, \mathrm{CH}_{3}$ ), 0.89 (d. $J=7.2 \mathrm{~Hz}, 3 \mathrm{H}$, $\mathrm{CH}_{3}$.

7-Bromo-4-hydroxy-2H-N-(5-isobutyl-3-aminohydantoinyl)-1,2-benzothiazine-3-carboxamide 1,1-dioxide $8 \mathrm{i}$. Yield $56 \%$. mp $297.298^{\circ} \mathrm{C}$ (dec), Recrystal solvent: methanol: IR $\left(\mathrm{NaCl}, \mathrm{cm}^{-1}\right) 3295(\mathrm{NH}), 3130(\mathrm{NH}), 3010$ (aromatic), $1720(\mathrm{CO}), 1475\left(\mathrm{SO}_{2}\right) .720(\mathrm{C} \cdot \mathrm{Br}){ }^{1} \mathrm{H}$ NMR (DMSO-d 6$)$ 11.08 (s, 1H. NH), 8.68 (s. IH. OH), 8.09-7.95 (m, 3H, $\mathrm{BrC}_{6} \mathrm{H}_{3}$ ). $4.30(\mathrm{t} . J=5.4 \mathrm{~Hz} . \mathrm{lH}, \mathrm{CH}), 1.84-1.82(\mathrm{~m} . \mathrm{lH}$. $\mathrm{CH}), 1.60-1.49\left(\mathrm{~m} .2 \mathrm{H}, \mathrm{CH}_{2}\right) .0 .92(\mathrm{~d}, J=7.2 \mathrm{~Hz} .6 \mathrm{H}$, $\mathrm{CH}_{2} \times 2$ ).

Acknowledgment. This work was supported by the research fund of Duksung Women s University

\section{References}

1. Braun. J. Chem. Ber. 1923, 56, 2332

2. Abe. K.: Yammamoto. S.: Matsui. K. Yakagaku zasshi 1956. 76. 1058: Chem. Atsstr. 1957.51.3499.

3. Soler. J. E. L. S. Paten 1985. 4.563.452

4. Suzuli. A.: Yajima, Y.: Yokoyama, S.: Matsuoka, Y.: Irimajiri, S. Japan Phannacol. Then 1994, 22. 221: Marfat. A. Amual Drug Data wapont 1987, p 202: U. S. Patem 1985, 4.551,452.

5. Turck. D.: Busch. U.: Heinzel. G.: Narjes. H.: Nehmiz. G. Chm. Drug hnest. 1995. 9. 270): Engelhardt. G. Brit. J. Rhetmutol. 1995. 3+. 90 .

6. Caruso. I: Montrone, F.: Boari. L. Ad Ther. 1994. 11, 132

7. Lombardino. J. G.: Wiseman, E. H.; Mclamore. W. d. Mfed. Chem. 1971. 14,1171 .

8. Kwon. S. K.: Park. M. S. Arznein-Forsch Drug Res. 1996. f6(II). 966.

9. Park. M. S. J. Korew Chent. Soc. 1998. +2(6). 657.

10. Kwon. S. K.: Park, M. S. Bull. Konew Chem. Soc. 1992, 13(5). 526.

11. Lalezan, I. J. Heterocychic. Chem. 1985. 22.741

12. Kwon. S. K.: Jung. H. W.: Park. M. S. Korean J of Med Chem. 1995. $5(2) .166$ 